\title{
Constraining the gluon PDF at large $x$ with LHC data
}

\author{
Emanuele R. Nocera*t \\ Rudolf Peierls Centre for Theoretical Physics, University of Oxford \\ 1 Keble Road, OXI 3NP Oxford, United Kingdom \\ E-mail: emanuele.nocera@physics.ox.ac.uk \\ Maria Ubiali* ${ }^{\dagger}$ \\ Cavendish Laboratory, HEP Group, University of Cambridge \\ 19 J.J. Thomson Avenue, CB3 OHE Cambridge, United Kingdom \\ E-mail: ubiali@hep.phy.cam.ac.uk
}

\begin{abstract}
The NNPDF collaboration has recently presented NNPDF3.1, a new determination of the parton distribution functions (PDFs) of the proton including a number of new data, some of which are particularly sensitive to the gluon PDF at large $x$. In this contribution, we present the results of two new analyses based on NNPDF3.1. First, we study the impact of the ATLAS $20117 \mathrm{TeV}$ inclusive jet data on the gluon PDF including different rapidity bins. Second, we quantitatively compare the constraints provided by all measurements sensitive to the large- $x$ gluon PDF, namely inclusive jet cross sections, the transverse momentum distributions of the $Z$-boson and top-pair rapidity distributions.
\end{abstract}

XXV International Workshop on Deep-Inelastic Scattering and Related Subjects

3-7 April 2017

University of Birmingham, $U K$

\footnotetext{
* Speaker.

$\dagger$ Joint contribution.
} 
Introduction. In a recent paper [1] the NNPDF Collaboration presented NNPDF3.1, a new set of parton distribution functions (PDFs) [2] which features several improvements with respect to the previous global analysis, NNPDF3.0 [3], both in terms of methodology and data. On the methodological side, the charm PDF was parametrised on the same footing as the other light quark and gluon PDFs. On the data side, a number of new measurements, especially from Large Hadron Collider (LHC) experiments, were included. Among these, some are directly sensitive to the gluon PDF at medium-to-large values of Bjorken $x$, which allowed us to determine it with a much improved precision. This is a remarkable feature of NNPDF3.1, as a detailed knowledge of the gluon PDF at large $x$ is increasingly crucial in order to generate precise predictions of both the signal and the backgrounds in searches for new massive particles at the LHC.

In this contribution we present two new analyses that were not included in NNPDF3.1. First, we study the stability of the NNPDF3.1 fit upon the inclusion of either of the five bins in which the inclusive $7 \mathrm{TeV}$ ATLAS 2011 jet data [4] are provided. We demonstrate that the particular choice made in the default NNDPF3.1 fit, i.e. the central rapidity bin, does not affect the ensuing gluon PDF. Second, we provide a quantitative comparison among the constraints provided by the three different datasets included in NNDPF3.1 that are sensitive to the gluon PDF at large $x$ : inclusive jet cross section, $Z$-boson transverse momentum distribution, and top rapidity distribution data. We make explicit the impact of each of these observables by adding the corresponding datasets, one at a time, to a baseline dataset that does not include any of them. This is different from what was presented in the NNPDF3.1 paper, in which one dataset at a time was removed from the global fit.

Stability of NNPDF3.1 upon the choice of rapidity bin for the ATLAS 2011 inclusive jet data. The NNPDF3.1 analysis included for the first time the single-inclusive jet cross sections measured in the 2011 run at $7 \mathrm{TeV}$ with $R=0.6$ by ATLAS and at $2.76 \mathrm{TeV}$ with $R=0.7$ by CMS. These were added on top of four measurements already included in NNPDF3.0, namely: CDF Run II kT, CMS 2011, 2010 ATLAS $7 \mathrm{TeV}$ and ATLAS $2.76 \mathrm{TeV}$, including correlations to the $7 \mathrm{TeV}$ data (see [1] for the experimental references).

Although next-to-next-to-leading order (NNLO) corrections to the inclusive jet production cross section are now known [5] (in the leading-colour approximation), the exact results are not yet available for all jet datasets included in NNPDF3.1. Therefore, they were included in the NNPDF3.1 NNLO PDF fit using NNLO PDF evolution but next-to-leading order (NLO) matrix elements. A fully correlated theoretical systematic uncertainty, accounting for the missing higher order corrections in the matrix element, was added to the covariance matrix. We also note that the sign and the size of the NNLO corrections strongly depend on the central scale used in the predictions. If the jet transverse momentum $p_{T}$ is taken as the central scale, the NNLO/NLO $K$ factors vary between $-5 \%$ and $+10 \%$ in the range measured at the LHC, $100 \mathrm{GeV} \lesssim p_{T} \lesssim 2 \mathrm{TeV}$ [6].

While no cuts were applied to all jet datasets included in NNPDF3.1, for the 2011 ATLAS 7 $\mathrm{TeV}$ dataset a good agreement between data and theory was obtained when fitting only the central rapidity bin, $\left|y_{\text {jet }}\right|<0.5$. Concurrently, it was found that achieving a good description of the ATLAS $20117 \mathrm{TeV}$ dataset would be impossible, if all five rapidity bins were included simultaneously and if all cross-correlations among rapidity bins were taken into account accordingly. Such behaviour is most likely due to underestimated correlated uncertainties, whether they are of theoretical or of experimental nature, however the exact origin is not clear yet. Given the situation, it is important 


\begin{tabular}{lccc}
\hline Fit & $\chi_{\text {ATLAS }}^{2} / N_{\text {dat }}($ before fit $)$ & $\chi_{\text {ATLAS }}^{2} / N_{\text {dat }}($ after fit $)$ & $\chi_{\text {tot }}^{2} / N_{\text {dat }}($ after fit $)$ \\
\hline NNPDF3.1 centralbin & 1.07 & 1.07 & 1.148 \\
NNPDF3.1 bin2 & 1.27 & 1.27 & 1.150 \\
NNPDF3.1 bin3 & 0.95 & 0.93 & 1.151 \\
NNPDF3.1 bin4 & 1.06 & 1.07 & 1.145 \\
NNPDF3.1 bin5 & 0.97 & 0.96 & 1.146 \\
NNPDF3.1 bin6 & 0.73 & 0.67 & 1.145 \\
\hline
\end{tabular}

Table 1: The $\chi^{2}$ per data point, $\chi^{2} / N_{\text {dat }}$, for the 2011 ATLAS $7 \mathrm{TeV}$ data set, before and after each variant of the default fit, and for the total data set after the fits. The first fit is the default NNPDF3.1 NNLO fit with $\alpha_{s}\left(M_{Z}\right)=0.118$, in which only the central jet rapidity bin $\left(\left|y_{\text {jet }}\right|<0.5\right)$ is included. In the following fits the central bin is replaced by the second, third, fourth, fifth and sixth rapidity bin respectively.

to demonstrate that the gluon PDF is stable upon the choice of any of the other rapidity bins. In order to investigate on this, we have performed five additional fits, with the same theoretical settings of the default NNPDF3.1 NNLO PDF fit, in which we have included in turn the second, third, fourth, fifth and sixth jet rapidity bin $\left(0.5<\left|y_{\text {jet }}\right|<1.0,1.0<\left|y_{\text {jet }}\right|<1.5,1.5<\left|y_{\text {jet }}\right|<2.0\right.$, $2.0<\left|y_{\text {jet }}\right|<2.5$ and $2.5<\left|y_{\text {jet }}\right|<3.0$ respectively) instead of the central bin.

In Table 1, we report the value of the $\chi^{2}$ per data point, $\chi^{2} / N_{\text {dat }}$, for the individual 2011 ATLAS $7 \mathrm{TeV}$ data set, before and after each of the five variants of the default fit, and for the total data set after the fits. In Fig. 1, we show the distance [7] between the central value and the uncertainty of the gluon PDF at $Q=100 \mathrm{GeV}$ in the default NNLO NNPDF3.1 fit and in each of the five variants of the fit including higher rapidity bins. All sets are made of $N_{\text {rep }}=100$ replicas. Distances of $d \simeq 1$ correspond to statistically equivalent fits, while for sets of 100 replicas $d \simeq 10$ corresponds to a difference of one sigma in unity of the corresponding variance. In Fig. 2 we compare the NNLO gluon PDF obtained from the NNPDF3.1 default fit and the variants in which the second and third rapidity bins of the 2011 ATLAS $7 \mathrm{TeV}$ jet data are fitted instead of the central bin. Very similar plots are found for fits with higher rapidity bins, therefore they are not shown. As is apparent from Table 1 and Figs. 1-2, the description of each separate bin is equally good, the central values of the gluon PDF are well within its uncertainty for each fit and PDFs are statistically equivalent. Therefore, we conclude that the gluon PDF in the NNPDF3.1 set is independent of the choice of the ATLAS 2011 jet data bin used in the fit.

Impact of various datasets on the gluon PDF at large $x$. On top of the inclusive jet data, the two main observables sensitive to the medium-to-large $x$ gluon PDF, that were included in NNDPF3.1, are the total rate and differential distributions of top-pair production and the transverse momentum distributions of the $Z$ boson. All of them have been measured by the ATLAS and CMS experiments at the LHC with high precision. The corresponding NNLO QCD corrections have been computed for top-pair production cross sections at the level of total rates in $[8,9,10]$ and of differential cross sections in [11, 12, 13, 14]; for the transverse momentum of the $Z$ boson in $[15,16,17]$. The impact on the gluon PDF of the newly available data sets for each of these observables has been studied in detail $[18,19,20,21]$. They have been shown to provide complementary and compatible constraints onto the gluon PDF at medium and large $x$, and that this constraint is competitive with that provided by inclusive jet data. 


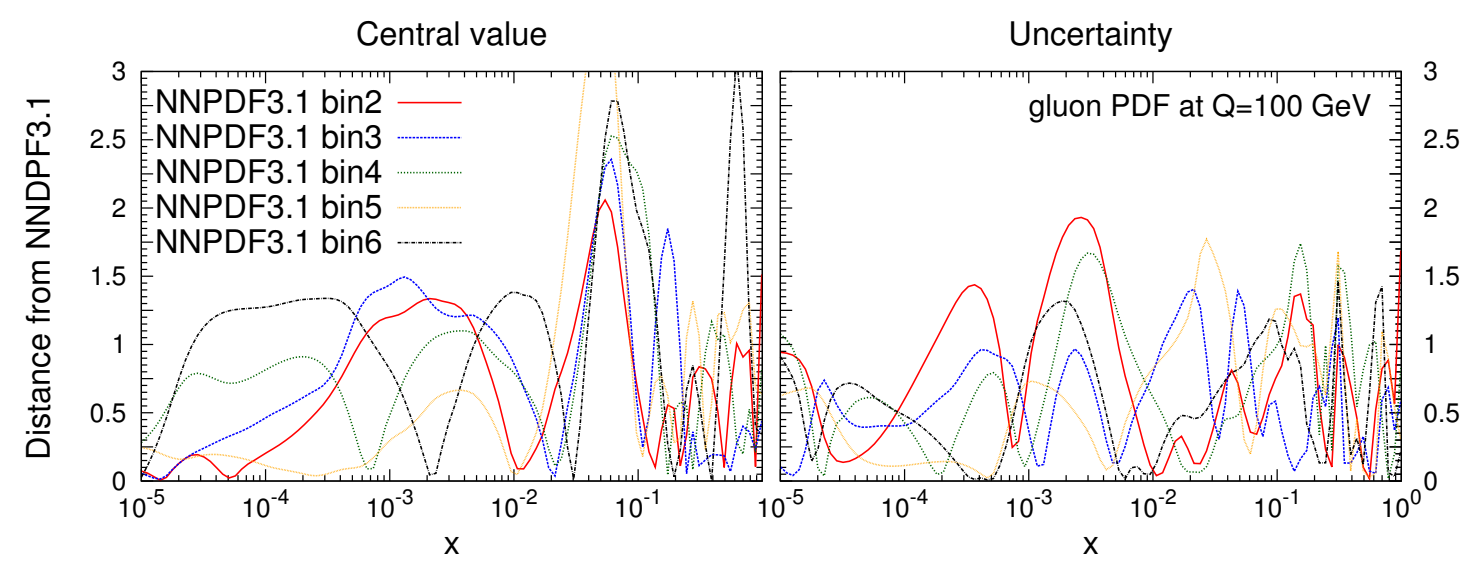

Figure 1: Distances between the central value (left) and the uncertainty (right) of the gluon PDF at $Q=100$ $\mathrm{GeV}$ in the NNPDF3.1 default NNLO fit and in the five variants in which higher jet rapidity bins are included instead of the central bin, see the text for details.
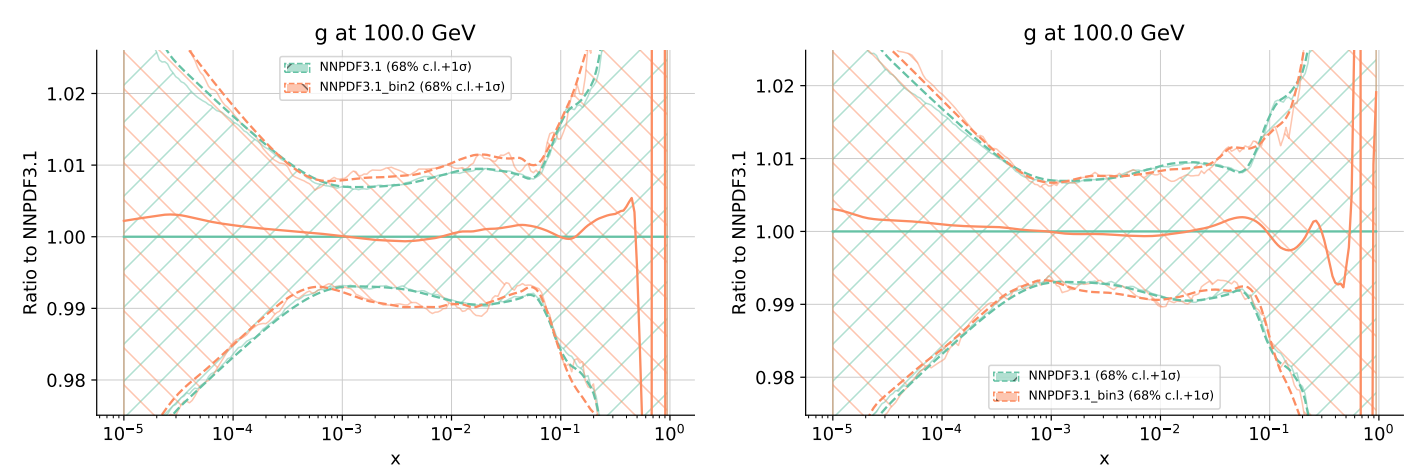

Figure 2: Comparison between the NNPDF3.1 default NNLO fit and two fits in which the second (left panel) and third (right panel) rapidity bin of the ATLAS $20117 \mathrm{TeV}$ data are included instead of the central bin. The factorisation scale is set to $Q=100 \mathrm{GeV}$ and PDFs are normalised to the NNPDF3.1 set.

Specifically, the new data sets directly sensitive to the gluon PDF at large $x$ that were included in NNPDF3.1 are the following: the $Z$ boson $\left(p_{T}^{Z}, y_{Z}\right)$ and $\left(p_{T}^{Z}, M_{l l}\right)$ double differential distributions at $8 \mathrm{TeV}$ from ATLAS and the $Z$ boson $\left(p_{T}^{Z}, y_{Z}\right)$ double differential distribution at $8 \mathrm{TeV}$ from CMS; the top-pair production normalised $y_{t}$ differential distribution at $8 \mathrm{TeV}$ from ATLAS, the top-pair production normalised $y_{t \bar{t}}$ differential distribution at $8 \mathrm{TeV}$ from CMS and the total inclusive cross sections for top-pair production at 7, 8 and $13 \mathrm{TeV}$ from ATLAS and CMS (for all references to the experimental papers, see [1]).

In NNPDF3.1, the three classes of measurements (inclusive jets, $Z p_{T}$ and top data) have all been included at the same time in the global fit. This was compared to variants in which either of the three measurements was removed at a time from the global dataset. This shows only indirectly the impact of each of these datasets. A much more direct comparison is the one in which each dataset is added individually to a baseline dataset made up of all the data in NNPDF3.1 except the piece sensitive to the gluon at large $x$. Therefore, we run four additional fits, with the same theoretical settings as in the NNPDF3.1 NNLO default fit. In the baseline fit, all jet, $Z p_{T}$ and 


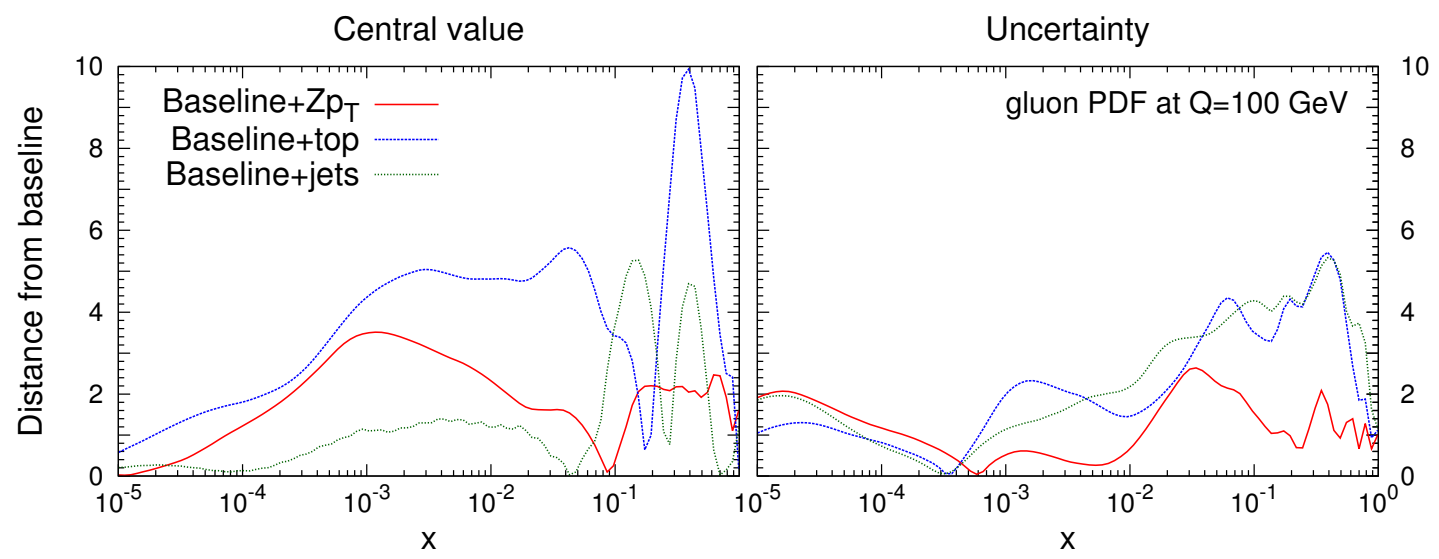

Figure 3: Distances between the central value (left) and the uncertainty (right) of the gluon PDF at $Q+100$ $\mathrm{GeV}$ in the NNPDF3.1 baseline NNLO fit without jet, $Z p_{T}$ and top data and the three variants in which each of these datasets is added at a time on top of the baseline.

top data are removed from the NNPDF3.1 NNLO fit. In the other three fits, we add each of these measurements individually on top of the baseline dataset. Notice that, in order to compare the impact of the three measurements as closely as possible, in the fit in which the jet data are added on top of the baseline data set, only the ATLAS and the CMS 2011 data at are included using the exact NNLO theory, which is available for these measurements. For a detailed discussion of the impact of the inclusion of jet inclusive data using the NNLO theory versus an NLO theory accounting for an extra theoretical uncertainty, see Ref. [1].

The distance in the central value and uncertainty between each of these three fits and the baseline is shown in Fig. 3. The corresponding gluon PDFs are compared in Fig. 4. At the level of the central value, we observe from Figs. 3-4 that the three sets of observables favour a slightly softer gluon PDF then the baseline above $x \sim 0.2$. Such an effect, up to half a $\sigma$, is more pronounced for jet and top data. At the same time, $Z$ transverse momentum and top data favour a slightly harder gluon between $10^{-2}$ and $10^{-1}$. At the level of uncertainties, the transverse momentum distribution of the $Z$ boson decreases the gluon PDF uncertainty by almost a factor of two for $10^{-2} \lesssim x \lesssim 10^{-1}$, while keeping the central value well within the baseline error band. Top-pair and jet production data have a bigger impact, as the relative uncertainty is reduced by almost $100 \%$ for all $x \gtrsim 10^{-2}$. All data sets consistently pull the gluon central value in the same direction; most noteworthily, top data provide a constraint on it competitive with inclusive jet data.

In summary, the combined effect of $Z p_{T}$, top and jet data is to consistently constrain the gluon PDF at medium-to-large values of $x$ with unprecedented, few percent, precision. Such a level of precision in the knowledge of the large- $x$ gluon is a remarkable achievement of the LHC experimental program, which can only further improve thanks to data at higher luminosity and centre-of-mass energy in the future.

We are grateful to our colleagues in the NNPDF Collaboration, especially to S. Carrazza, S. Forte, J. Rojo and L. Rottoli. E.R.N. is supported by the the STFC grant ST/M003787/1. M.U. is supported by a Royal Society Dorothy Hodgkin Research Fellowship and partially by the STFC grant ST/L000385/1. 

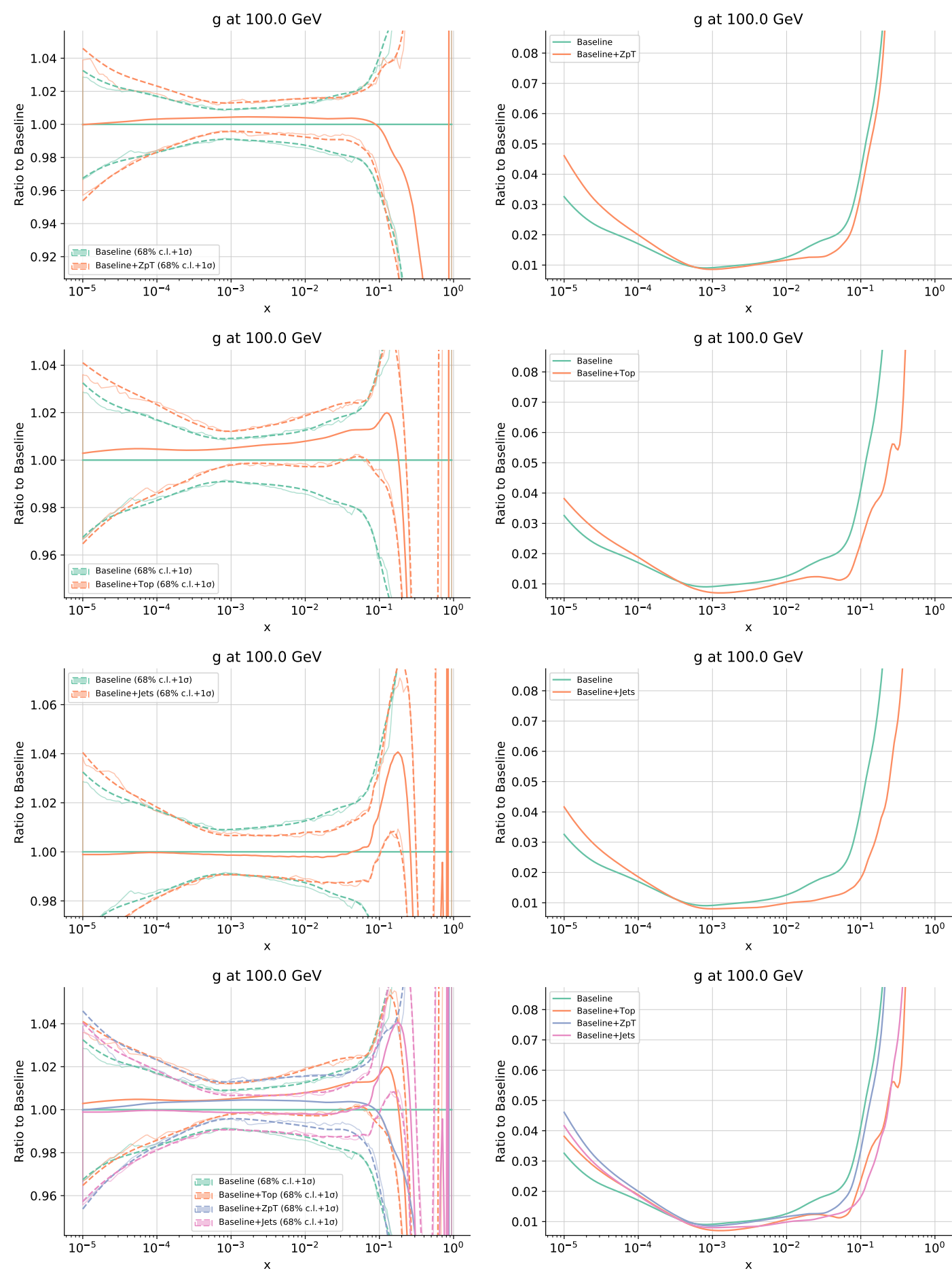

Figure 4: Comparison between the gluon PDF as obtained from a baseline fit that includes all the data in the NNDPF3.1 analysis except $Z p_{T}$, top and jet measurements, and three fits in which each of these data sets is included in turn on top of the baseline data set: $Z p_{T}$ measurements (first row), top differential distribution and total cross section measurements (second row) and inclusive jet cross section measurements by using the exact NNLO $K$-factors (third row). The results are displayed simultaneously in the fourth row. In the left panels, the gluon PDF is normalised to the baseline fit, in the right panel relative uncertainties are shown. The factorisation scale is set to $Q=100 \mathrm{GeV}$. 


\section{References}

[1] R. D. Ball et al. [NNPDF Collaboration], arXiv:1706.00428 [hep-ph].

[2] J. Gao, L. Harland-Lang and J. Rojo, arXiv:1709.04922 [hep-ph].

[3] R. D. Ball et al. [NNPDF Collaboration], JHEP 1504 (2015) 040.

[4] G. Aad et al. [ATLAS Collaboration], JHEP 1502 (2015) 153 Erratum: [JHEP 1509 (2015) 141].

[5] J. Currie, E. W. N. Glover and J. Pires, Phys. Rev. Lett. 118 (2017) 072002.

[6] J. Currie et al., Acta Phys. Polon. B 48 (2017) 955.

[7] R. D. Ball et al., Nucl. Phys. B 838 (2010) 136.

[8] M. Czakon, P. Fiedler and A. Mitov, Phys. Rev. Lett. 110 (2013) 252004.

[9] M. Czakon and A. Mitov, JHEP 1301 (2013) 080.

[10] P. Bärnreuther, M. Czakon and A. Mitov, Phys. Rev. Lett. 109 (2012) 132001.

[11] M. Czakon, D. Heymes and A. Mitov, Phys. Rev. Lett. 116 (2016) 082003.

[12] M. Czakon, P. Fiedler and A. Mitov, Phys. Rev. Lett. 115 (2015) 052001.

[13] M. Czakon, P. Fiedler, D. Heymes and A. Mitov, JHEP 1605 (2016) 034.

[14] M. Czakon, D. Heymes and A. Mitov, JHEP 1704 (2017) 071.

[15] R. Boughezal et al., Phys. Rev. Lett. 116 (2016) 152001.

[16] A. Gehrmann-De Ridder et al., JHEP 1607 (2016) 133.

[17] A. Gehrmann-De Ridder et al., JHEP 1611 (2016) 094.

[18] M. Beneke, P. Falgari, S. Klein, J. Piclum, C. Schwinn, M. Ubiali and F. Yan, JHEP 1207 (2012) 194.

[19] M. Czakon, M. L. Mangano, A. Mitov and J. Rojo, JHEP 1307 (2013) 167.

[20] M. Czakon, N. P. Hartland, A. Mitov, E. R. Nocera and J. Rojo, JHEP 1704 (2017) 044.

[21] R. Boughezal, A. Guffanti, F. Petriello and M. Ubiali, JHEP 1707 (2017) 130. 